\title{
SPECTRA OF WEIGHTED ROOTED GRAPHS HAVING PRESCRIBED SUBGRAPHS AT SOME LEVELS*
}

\author{
OSCAR ROJO ${ }^{\dagger}$, MARIA ROBBIANO $¥$, DOMINGOS M. CARDOSO ${ }^{\S}$, AND ENIDE A. \\ MARTINS $\S$
}

\begin{abstract}
Let $\mathcal{B}$ be a weighted generalized Bethe tree of $k$ levels $(k>1)$ in which $n_{j}$ is the number of vertices at the level $k-j+1(1 \leq j \leq k)$. Let $\Delta \subseteq\{1,2, \ldots, k-1\}$ and $F=\left\{\mathcal{G}_{j}: j \in \Delta\right\}$, where $\mathcal{G}_{j}$ is a prescribed weighted graph on each set of children of $\mathcal{B}$ at the level $k-j+1$. In this paper, the eigenvalues of a block symmetric tridiagonal matrix of order $n_{1}+n_{2}+\cdots+n_{k}$ are characterized as the eigenvalues of symmetric tridiagonal matrices of order $j, 1 \leq j \leq k$, easily constructed from the degrees of the vertices, the weights of the edges, and the eigenvalues of the matrices associated to the family of graphs $F$. These results are applied to characterize the eigenvalues of the Laplacian matrix, including their multiplicities, of the graph $\mathcal{B}(F)$ obtained from $\mathcal{B}$ and all the graphs in $F=\left\{\mathcal{G}_{j}: j \in \Delta\right\}$; and also of the signless Laplacian and adjacency matrices whenever the graphs of the family $F$ are regular.
\end{abstract}

Key words. Weighted graph, Laplacian matrix, Signless Laplacian matrix, Adjacency matrix, Generalized Bethe tree.

AMS subject classifications. 05C50, 15A18.

1. Introduction. Let $\mathcal{G}=(V, E)$ be a simple undirected graph with vertex set $V$ and edge set $E$. We assume that each edge $e \in E$ has a positive weight $w(e)$. Let $V=\{1,2, \ldots, n\}$. The Laplacian matrix $L(\mathcal{G})=\left(l_{i, j}\right)$, the signless Laplacian matrix $Q(\mathcal{G})=\left(q_{i, j}\right)$ and the adjacency matrix $A(\mathcal{G})=\left(a_{i, j}\right)$ of the graph $\mathcal{G}$, are the $n \times n$ matrices defined by

$$
l_{i, j}=\left\{\begin{array}{cl}
-w(e) & \text { if } i \neq j \text { and } e \text { is the edge joining } i \text { and } j \\
0 & \text { if } i \neq j \text { and } i \text { is not adjacent to } j \\
-\sum_{k \neq i} l_{i, k} & \text { if } i=j
\end{array}\right.
$$

*Received by the editors on November 24, 2010. Accepted for publication on May 20, 2011. Handling Editor: Stephen J. Kirkland.

†Departamento de Matemáticas, Universidad Católica del Norte, Antofagasta, Chile (orojo@ucn.cl). Research supported by Project Fondecyt Regular 1100072, Chile.

${ }^{\ddagger}$ Departamento de Matemáticas, Universidad Católica del Norte, Antofagasta, Chile (mrobbiano@ucn.cl). Research partially supported by Fondecyt - IC Project 11090211, Chile.

$\S$ Departamento de Matemática, Universidade de Aveiro, Aveiro, Portugal (dcardoso@ua.pt, enide@ua.pt). Research supported by the Centre for Research and Development in Mathematics and Applications from the Fundação para a Ciência e a Tecnologia - FCT (cofinanced by European Community Fund FEDER/POCI 2010) and project PTDC/MAT/112276/2009. 


$$
\begin{aligned}
& q_{i, j}=\left\{\begin{array}{cc}
w(e) & \text { if } i \neq j \text { and } e \text { is the edge joining } i \text { and } j \\
0 & \text { if } i \neq j \text { and } i \text { is not adjacent to } j \\
\sum_{k \neq i} q_{i, k} & \text { if } i=j
\end{array},\right. \text { and } \\
& a_{i, j}=\left\{\begin{array}{cl}
w(e) & \text { if } i \neq j \text { and } e \text { is the edge joining } i \text { and } j \\
0 & \text { if } i \neq j \text { and } i \text { is not adjacent to } j \\
0 & \text { if } i=j
\end{array}\right.
\end{aligned}
$$

Then $L(\mathcal{G}), Q(\mathcal{G})$ and $A(\mathcal{G})$ are real symmetric matrices. From Geršgorin's Theorem, it follows that the eigenvalues of $L(\mathcal{G})$ and $Q(\mathcal{G})$ are nonnegative real numbers. Since the sum of the entries in each row of $L(\mathcal{G})$ is 0 , then $(0, \mathbf{e})$ is an eigenpair for $L(\mathcal{G})$ where $\mathbf{e}$ is the all ones vector. Fiedler [8] proved that $\mathcal{G}$ is a connected graph if and only if the second smallest eigenvalue of $L(\mathcal{G})$ is positive. This eigenvalue, denoted by $a(\mathcal{G})$, is called the algebraic connectivity of $\mathcal{G}$. The signless Laplacian matrix has attracted the attention of several researchers. Recent papers on spectral results with this matrix are $[1,3,4,5,6]$.

If $w(e)=1$ for all $e \in E$, then $\mathcal{G}$ is an unweighted graph. In [10], some of the known results for the Laplacian matrix of an unweighted graph are given.

We recall that for a rooted graph, the level of a vertex is one more than its distance from the root vertex.

A generalized Bethe tree is a rooted tree in which vertices at the same level have the same degree. Throughout this paper, $\mathcal{B}=\mathcal{B}_{k}(d)$ is a generalized Bethe tree with $k$ levels $(k>1)$, such that $d$ is a $k$-tuple, where the $j$ th entry $d_{j}$ is the degree of the vertices at level $k-j+1$, in which the edges connecting vertices at consecutive levels have the same weight. In $\mathcal{B}$, for $1 \leq j \leq k, n_{j}$ denotes the number of vertices at the level $k-j+1$. Therefore, $d_{k}$ is the degree of the root vertex, $n_{k}=1, d_{1}=1$, and $n_{1}$ is the number of pendant vertices. We assume that for the vertex at level 1 , that is, for the root vertex, we have $d_{k}>1$.

For $1 \leq j \leq k-1, w_{j}$ is the weight of the edges connecting the vertices of $\mathcal{B}$ at the level $k-j+1$ with the vertices at the level $k-j$. Furthermore, we define

$$
\delta_{j}= \begin{cases}w_{1} & \text { if } j=1 \\ \left(d_{j}-1\right) w_{j-1}+w_{j} & \text { if } 2 \leq j \leq k-1 \\ d_{k} w_{k-1} & \text { if } j=k\end{cases}
$$

Observe that $\delta_{j}$ is the sum of the weights of the edges of $\mathcal{B}$ incident with the vertices of $\mathcal{B}$ at the level $k-j+1$ and if $w_{1}=w_{2}=\cdots=w_{k-1}=1$, then $\delta_{j}=d_{j}$ for $j=1, \ldots, k$.

Setting $m_{j}=\frac{n_{j}}{n_{j+1}}$ for $j=1, \ldots, k-1$, it follows

$$
m_{j}=d_{j+1}-1 \quad(1 \leq j \leq k-2)
$$




$$
d_{k}=n_{k-1}=m_{k-1}
$$

Consider an index subset $\Delta \subseteq\{1,2, \ldots, k-1\}$, and a family of graphs $F=$ $\left\{\mathcal{G}_{j}: j \in \Delta\right\}$, where each graph $\mathcal{G}_{j}$ has order $m_{j}, j=1, \ldots, k$. Then we define $\mathcal{B}(F)$ as the graph obtained from $\mathcal{B}$ and the graphs in $F$ identifying each set of children of $\mathcal{B}$ at level $k-j+1$, with the vertices of $\mathcal{G}_{j}$, for $j \in \Delta$. We assume that the edges of $\mathcal{G}_{j}$ have a weight $u_{j}$. Since $\mathcal{B}$ and $\mathcal{B}(F)$ have the same set of vertices, we may label the vertices of $\mathcal{B}(F)$ from the pendant vertices to the root and, in each level, from the left to the right.

Throughout this paper, we denote by $\mathcal{P}_{m}(u), \mathcal{S}_{m}(u), \mathcal{C}_{m}(u)$, and $\mathcal{K}_{m}(u)$ the path, star, cycle, and complete graph on $m$ vertices, respectively, in which all the edges have weight equal to $u$.

ExAmPle 1.1. Let $\mathcal{B}=\mathcal{B}_{4}(1,5,3,2), \Delta=\{1,3\}$ and consider the family of graphs $F=\left\{\mathcal{G}_{1}=\mathcal{S}_{4}\left(u_{1}\right), \mathcal{G}_{3}=\mathcal{P}_{2}\left(u_{3}\right)\right\}$. Then $\mathcal{B}(F)$ is the graph depicted in Figure 1.1 .

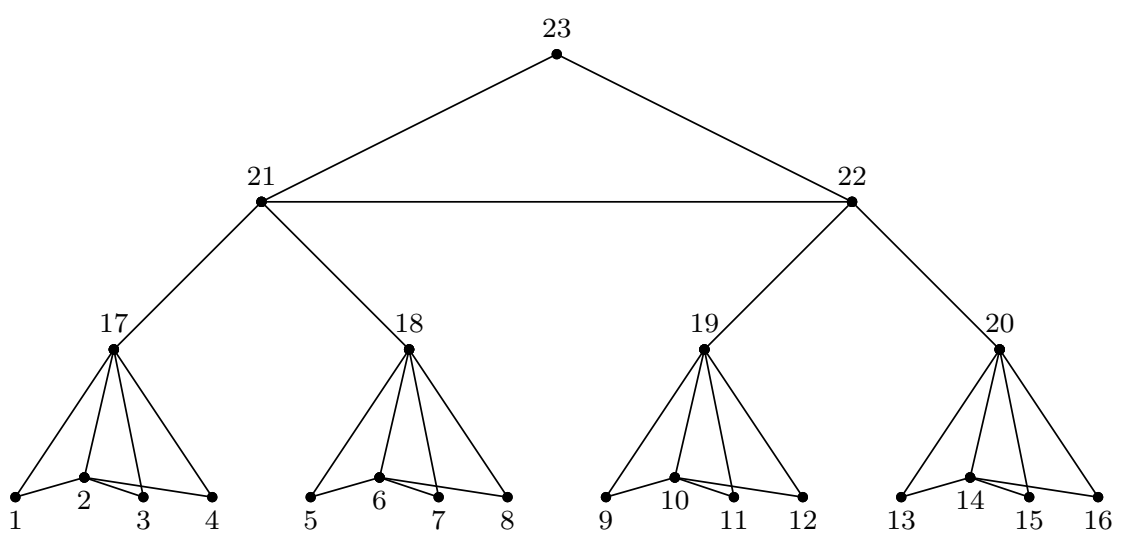

FIG. 1.1. Graph $\mathcal{B}(\mathrm{F})$, with $F=\left\{\mathcal{G}_{1}=\mathcal{S}_{4}\left(u_{1}\right), \mathcal{G}_{3}=\mathcal{P}_{2}\left(u_{3}\right)\right\}$.

Observe that since $\mathcal{B}(F)$ has $k=4$ levels and $\Delta=\{1,3\}$, then for $j=1$, at level $4-1+1=4$, there are four graphs $\mathcal{G}_{1}=\mathcal{S}_{4}\left(u_{1}\right)$, each one with vertex set defined by the set of children of a vertex at level 3 and, for $j=3$, at level $4-3+1=2$, there is one graph $\mathcal{G}_{3}=\mathcal{P}_{2}\left(u_{3}\right)$ with vertex set defined by the unique set of children of the root vertex.

Throughout this text, the identity matrix of appropriate order is denoted by $I$ and $I_{m}$ denotes the identity matrix of order $m$.

We recall that the Kronecker product (cf. [13]) of two matrices $A=\left(a_{i, j}\right)$ and $B=\left(b_{i, j}\right)$ of sizes $m \times m$ and $n \times n$, respectively, is defined as the $(m n) \times(m n)$ matrix 
$A \otimes B=\left(a_{i, j} B\right)$. Then, in particular, $I_{n} \otimes I_{m}=I_{n m}$.

Some basic properties of the Kronecker product are the following:

$$
(A \otimes B)^{T}=A^{T} \otimes B^{T}
$$

and

$$
(A \otimes B)(C \otimes D)=(A C \otimes B D)
$$

for matrices of appropriate sizes. Moreover, if $A$ and $B$ are invertible matrices, then

$$
(A \otimes B)^{-1}=A^{-1} \otimes B^{-1}
$$

Furthermore, we need the following additional notation: $|A|$ denotes the determinant of the matrix $A$, when $A$ is square and, for any matrix $B, B^{T}$ denotes the transpose of $B$.

In this paper, we characterize the eigenvalues of the matrix $S=$

$$
\left[\begin{array}{ccccc}
I_{n_{2}} \otimes S_{1} & \mp I_{n_{2}} \otimes w_{1} \mathbf{e}_{m_{1}} & & & \\
\mp I_{n_{2}} \otimes w_{1} \mathbf{e}_{m_{1}}^{T} & \ddots & \ddots & & \\
& \ddots & I_{n_{k-1}} \otimes S_{k-2} & \mp I_{n_{k-1}} \otimes w_{k-2} \mathbf{e}_{m_{k-2}} & \\
& & \mp I_{n_{k-1}} \otimes w_{k-2} \mathbf{e}_{m_{k-2}}^{T} & S_{k-1} & \mp w_{k-1} \mathbf{e}_{m_{k-1}} \\
& & & \mp w_{k-1} \mathbf{e}_{m_{k-1}}^{T} & \gamma_{k}
\end{array}\right],
$$

where

$$
S_{j}=\gamma_{j} I_{m_{j}}+c_{j} M_{j} \quad(1 \leq j \leq k-1)
$$

$\gamma_{j}$ and $c_{j}$ are scalars, and $M_{j}$ is a matrix on the graph $\mathcal{G}_{j}$, having $\mathbf{e}_{m_{j}}$ as one of its eigenvector, if $j \in \Delta$ and it is the zero matrix otherwise.

For instance, if

$$
L_{j}=\left\{\begin{array}{ll}
\delta_{j} I_{m_{j}}+L\left(\mathcal{G}_{j}\right) & \text { if } j \in \Delta \\
\delta_{j} I_{m_{j}} & \text { otherwise }
\end{array}, \quad j=1, \ldots, k-1,\right.
$$

then, using the above mentioned labelling for the vertices, the Laplacian matrix of $\mathcal{B}(F)$ is $L(\mathcal{B}(F))=$

$$
\left[\begin{array}{ccccc}
I_{n_{2}} \otimes L_{1} & -I_{n_{2}} \otimes w_{1} \mathbf{e}_{m_{1}} & & & \\
-I_{n_{2}} \otimes w_{1} \mathbf{e}_{m_{1}}^{T} & \ddots & \ddots & & \\
& \ddots & I_{n_{k-1}} \otimes L_{k-2} & -I_{n_{k-1}} \otimes w_{k-2} \mathbf{e}_{m_{k-2}} & \\
& & -I_{n_{k-1}} \otimes w_{k-2} \mathbf{e}_{m_{k-2}}^{T} & L_{k-1} & -w_{k-1} \mathbf{e}_{m_{k-1}} \\
& & & -w_{k-1} \mathbf{e}_{m_{k-1}}^{T} & \delta_{k}
\end{array}\right]
$$


We prove that the eigenvalues of $S$ are the eigenvalues of a set of symmetric tridiagonal matrices of order $j, 1 \leq j \leq k$, easily constructed from the degrees of the vertices, the weights of the edges and the eigenvalues of the matrices $M_{j}$, which in some cases are very well known. Then, we apply these results to characterize the eigenvalues of the Laplacian (and also the signless Laplacian and adjacency matrices, when the graphs of the family $F$ are regular), including their multiplicities, of the graph $\mathcal{B}(F)$.

The results of this paper generalize the results of some previous works. Among them we may refer $[2,7,11]$. In [2], the authors characterize the eigenvalues of the Laplacian matrix for the unweighted case, whenever $\Delta=\{j\}$ and the graphs $\mathcal{G}_{j}$ are the paths $\mathcal{P}_{m_{j}}$. In [7], the authors characterize the eigenvalues of these matrices whenever, for $j \in \Delta=\left\{1 \leq j \leq k-1: m_{j}\right.$ even $\}$, the graphs $\mathcal{G}_{j}$ are the disconnected graphs $\frac{m_{j}}{2} \mathcal{P}_{2}$, that is, $\frac{m_{j}}{2}$ copies of the path $\mathcal{P}_{2}$. In [11], the eigenvalues of the Laplacian, signless Laplacian and adjacency matrices are characterized whenever, for each $j \in \Delta$, the graphs $\mathcal{G}_{j}$ are the complete graphs $\mathcal{K}_{m_{j}}$.

Throughout the next sections, 0 denotes the all zero matrix of appropriate order and $\mathbf{e}_{m}$ is the $m$-dimensional column vector of ones. Furthermore, the scalars $c_{j}$ are such that $c_{j}=1$ if $j \in \Delta$ and $c_{j}=0$ if $j \notin \Delta$.

2. The main result. From now on, if $M_{j}$ is a matrix on the graph $\mathcal{G}_{j}$, with eigenvalues

$$
\mu_{1}\left(M_{j}\right), \ldots, \mu_{m_{j}}\left(M_{j}\right)
$$

then $\mu_{m_{j}}\left(M_{j}\right)$ is such that

$$
M_{j} \mathbf{e}_{m_{j}}=\mu_{m_{j}}\left(M_{j}\right) \mathbf{e}_{m_{j}}
$$

Before introducing the main result of this paper, we prove the following auxiliary lemmas.

Lemma 2.1. Consider the matrix

$$
B=\beta I_{m}-c M
$$

where $\beta$ and $c$ are scalars and the matrix $M$ has the eigenvalues $\mu_{1}(M), \ldots, \mu_{m}(M)$. Then

$$
|B|=\prod_{i=1}^{m}\left(\beta-c \mu_{i}(M)\right) .
$$


Moreover, if $\beta-c \mu_{i}(M) \neq 0$ for all $i$ and $M \mathbf{e}_{m}=\mu_{m}(M) \mathbf{e}_{m}$, then

$$
\mathbf{e}_{m}^{T} B^{-1} \mathbf{e}_{m}=\frac{m}{\beta-c \mu_{m}(M)} .
$$

Proof. Clearly the eigenvalues of $B$ are $\beta-c \mu_{i}(M)(1 \leq i \leq m)$ and thus (2.1) follows. Since $M \mathbf{e}_{m}=\mu_{m}(M) \mathbf{e}_{m}$, then $B \mathbf{e}_{m}=\left(\beta-c \mu_{m}(M)\right) \mathbf{e}_{m}$. Therefore, assuming that these eigenvalues are nonzero, $B$ is invertible, $B^{-1} \mathbf{e}_{m}=\frac{1}{\beta-c \mu_{m}(M)} \mathbf{e}_{m}$ and (2.2) follows.

LEMma 2.2. Consider the block tridiagonal matrix $T=$

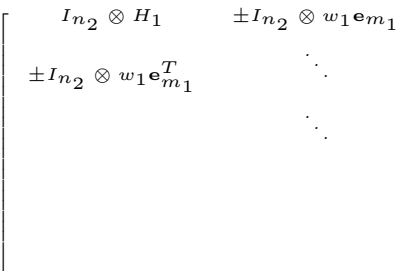

$$
\begin{gathered}
I_{n_{k-1}} \otimes H_{k-2} \\
\pm I_{n_{k-1}} \otimes w_{k-2} \mathbf{e}_{m_{k-2}}^{T}
\end{gathered}
$$
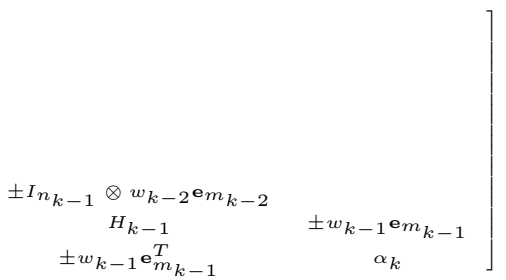

where, for $j=1, \ldots, k-1, H_{j}=\alpha_{j} I_{m_{j}}-c_{j} M_{j}$.

Assume

$$
\beta_{1}=\alpha_{1}
$$

and, for $j=2, \ldots, k$, assume also

$$
\beta_{j}=\alpha_{j}-\frac{w_{j-1}^{2} m_{j-1}}{\beta_{j-1}-c_{j-1} \mu_{m_{j-1}}\left(M_{j-1}\right)} .
$$

If $\beta_{j}-c_{j} \mu_{i}\left(M_{j}\right) \neq 0$ for $j=1, \ldots, k-1$ and $i=1, \ldots, m_{j}$, then

$$
|T|=\prod_{j=1}^{k} \prod_{i=1}^{m_{j}}\left(\beta_{j}-c_{j} \mu_{i}\left(M_{j}\right)\right)^{n_{j+1}} .
$$

Proof. In order to prove (2.3), we reduce $T$ to a block upper triangular matrix. We have $H_{1}=\alpha_{1} I_{m_{1}}-c_{1} M_{1}=\beta_{1} I_{m_{1}}-c_{1} M_{1} \equiv B_{1}$. From the hypothesis, it follows that $B_{1}$ is an invertible matrix. Multiplying the first row of blocks by $\pm I_{n_{2}} \otimes w_{1} \mathbf{e}_{m_{1}}^{T} B_{1}^{-1}$ and subtracting the products from the second row of blocks, we obtain $T_{2}=$

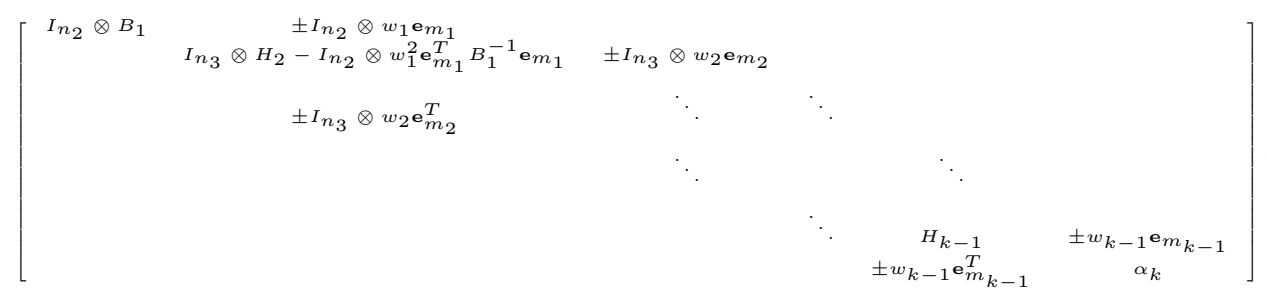


From Lemma 2.1, $\mathbf{e}_{m_{1}}^{T} B_{1}^{-1} \mathbf{e}_{m_{1}}=\frac{m_{1}}{\beta_{1}-c_{1} \mu_{m_{1}}\left(M_{1}\right)}$. Then

$$
\begin{aligned}
I_{n_{3}} \otimes H_{2}-I_{n_{2}} \otimes w_{1}^{2} \mathbf{e}_{m_{1}}^{T} B_{1}^{-1} \mathbf{e}_{m_{1}} & =I_{n_{3}} \otimes H_{2}-I_{n_{2}} \otimes \frac{w_{1}^{2} m_{1}}{\beta_{1}-c_{1} \mu_{m_{1}}\left(M_{1}\right)} \\
& =I_{n_{3}} \otimes H_{2}-\frac{w_{1}^{2} m_{1}}{\beta_{1}-c_{1} \mu_{m_{1}}\left(M_{1}\right)} I_{n_{3}} \otimes I_{m_{2}} \\
& =I_{n_{3}} \otimes\left(H_{2}-\frac{w_{1}^{2} m_{1}}{\beta_{1}-c_{1} \mu_{m_{1}}\left(M_{1}\right)} I_{m_{2}}\right) \\
& =I_{n_{3}} \otimes\left(\left(\alpha_{2}-\frac{w_{1}^{2} m_{1}}{\beta_{1}-c_{1} \mu_{m_{1}}\left(M_{1}\right)}\right) I_{m_{2}}-c_{2} M_{2}\right) \\
& =I_{n_{3}} \otimes\left(\beta_{2} I_{m_{2}}-c_{2} M_{2}\right) .
\end{aligned}
$$

If $B_{2}=\beta_{2} I_{m_{2}}-c_{2} M_{2}$, then $|T|=\left|T_{2}\right|=$

$$
\begin{array}{cccccc}
I_{n_{2}} \otimes B_{1} & \pm I_{n_{2}} \otimes w_{1} \mathbf{e}_{m_{1}} & \multicolumn{1}{l}{} \\
{ }_{0} & I_{n_{3}} \otimes B_{2} & \pm I_{n_{3}} \otimes w_{2} \mathbf{e}_{m_{2}} & & & \\
& \pm I_{n_{3}} \otimes w_{2} \mathbf{e}_{m_{2}}^{T} & \ddots & \ddots & & \\
& & \ddots & I_{n_{k-1}} \otimes H_{k-2} & \pm I_{n_{k-1}} \otimes w_{k-2} \mathbf{e}_{m_{k-2}} & \\
& & & H_{k-1} & \pm w_{k-1} \mathbf{e}_{m_{k-1}} \\
& & & \pm I_{n_{k-1}} \otimes w_{k-2} \mathbf{e}_{m_{k-2}}^{T} & \pm w_{k-1} \mathbf{e}_{m_{k-1}}^{T} & \alpha_{k}
\end{array} \mid
$$

From the hypothesis, the matrix $B_{2}$ is invertible and thus we may continue with this procedure. Just before the last step, we obtain $|T|=\left|T_{k-1}\right|=$

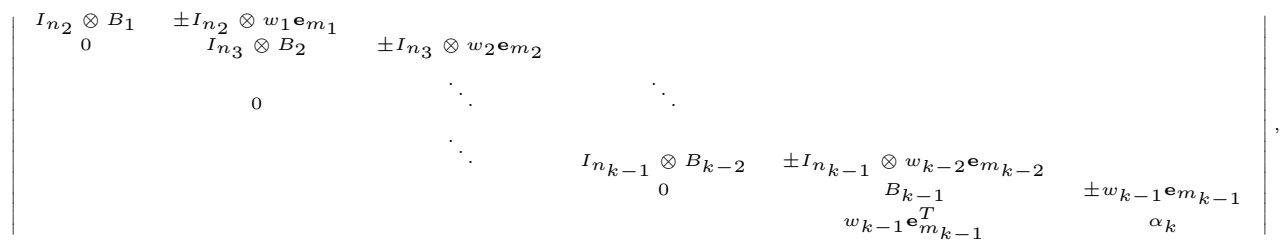

where $B_{j}=\beta_{j} I_{m_{j}}-c_{j} M_{j}, j=1, \ldots, k-1$. Finally, the procedure gives $|T|=$

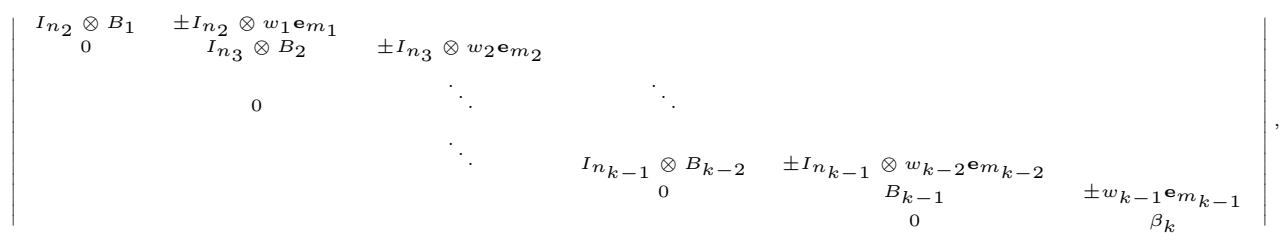

with $\beta_{k}=\alpha_{k}-\frac{w_{k-1}^{2} m_{k-1}}{\beta_{k-1}-c_{k-1} \mu_{m_{k-1}}\left(M_{k-1}\right)}$. Hence,

$$
|T|=\beta_{k} \prod_{j=1}^{k-1}\left|B_{j}\right|^{n_{j+1}} .
$$


From Lemma 2.1

$$
\left|B_{j}\right|=\prod_{i=1}^{m_{j}}\left(\beta_{j}-c_{j} \mu_{i}\left(M_{j}\right)\right) .
$$

Replacing in (2.4), we obtain (2.3).

Definition 2.3. The polynomials $D_{j}(\lambda), j=1, \ldots, k$ are defined as follows:

$$
\begin{gathered}
D_{0}(\lambda)=1 \\
D_{1}(\lambda)=\lambda-\gamma_{1}-c_{1} \mu_{m_{1}}\left(M_{1}\right) \\
D_{j}(\lambda)=\left(\lambda-\gamma_{j}-c_{j} \mu_{m_{j}}\left(M_{j}\right)\right) D_{j-1}(\lambda)-w_{j-1}^{2} m_{j-1} D_{j-2}(\lambda)
\end{gathered}
$$

for $j=2, \ldots, k-1$, and

$$
D_{k}(\lambda)=\left(\lambda-\gamma_{k}\right) D_{k-1}(\lambda)-w_{k-1}^{2} m_{k-1} D_{k-2}(\lambda)
$$

For brevity, we write $D_{j}$ instead of $D_{j}(\lambda)$.

LEMMA 2.4. If $\alpha_{j}=\lambda-\gamma_{j}$, for $j=1, \ldots, k$, then for all $\lambda \in \mathbb{R}$ such that $D_{j}(\lambda) \neq 0$, for $j=1, \ldots, k-1$, the quantities $\beta_{j}$ defined in Lemma 2.2 satisfy

$$
\beta_{j}-c_{j} \mu_{m_{j}}\left(M_{j}\right)=\frac{D_{j}}{D_{j-1}}
$$

for $j=1, \ldots, k$.

Proof. We prove (2.5) by induction on $j$, taking into account that for $j=1$,

$$
\begin{aligned}
\beta_{1}-c_{1} \mu_{m_{1}}\left(M_{1}\right) & =\alpha_{1}-c_{1} \mu_{m_{1}}\left(M_{1}\right)=\lambda-\gamma_{1}-c_{1} \mu_{m_{1}}\left(M_{1}\right) \\
& =D_{1}=\frac{D_{1}}{D_{0}} .
\end{aligned}
$$

Suppose that (2.5) is true for $j-1$, with $2 \leq j \leq k$. Then

$$
\begin{aligned}
\beta_{j}-c_{j} \mu_{m_{j}}\left(M_{j}\right) & =\alpha_{j}-\frac{w_{j-1}^{2} m_{j-1}}{\beta_{j-1}-c_{j-1} \mu_{m_{j-1}}\left(M_{j-1}\right)}-c_{j} \mu_{m_{j}}\left(M_{j}\right) \\
& =\lambda-\gamma_{j}-c_{j} \mu_{m_{j}}\left(M_{j}\right)-\frac{w_{j-1}^{2} m_{j-1}}{\frac{D_{j-1}}{D_{j-2}}} \\
& =\frac{\left(\lambda-\gamma_{j}-c_{j} \mu_{m_{j}}\left(M_{j}\right)\right) D_{j-1}-w_{j-1}^{2} m_{j-1} D_{j-2}}{D_{j-1}}=\frac{D_{j}}{D_{j-1}} .
\end{aligned}
$$


For convenience, from now on, let us define the index set

$$
\Omega=\left\{j: 1 \leq j \leq k-1, n_{j}>n_{j+1}\right\} .
$$

THEOREM 2.5. The characteristic polynomial of the matrix $S$ is

$$
\begin{aligned}
|\lambda I-S|=D_{k} & \prod_{j \in \Omega-\Delta}\left(\left(\lambda-\gamma_{j}\right) D_{j-1}-w_{j-1}^{2} m_{j-1} D_{j-2}\right)^{n_{j}-n_{j+1}} \\
& \prod_{j \in \Delta} \prod_{i=1}^{m_{j}-1}\left(\left(\lambda-\gamma_{j}-\mu_{i}\left(M_{j}\right)\right) D_{j-1}(\lambda)-w_{j-1}^{2} m_{j-1} D_{j-2}(\lambda)\right)^{n_{j+1}}
\end{aligned}
$$

where $m_{0}=0$ and $D_{-1}(\lambda)$ is any constant (since it appears multiplied by $m_{0}$ ).

Proof. Let $\lambda \in \mathbb{R}$ such that $D_{j}(\lambda) \neq 0$ for $j=1,2, \ldots, k-1$. Applying Lemma 2.2 to the matrix $T=\lambda I-S$, we have

$$
|\lambda I-S|=\beta_{k} \prod_{j=1}^{k-1} \prod_{i=1}^{m_{j}}\left(\beta_{j}-c_{j} \mu_{i}\left(M_{j}\right)\right)^{n_{j+1}} .
$$

Replacing (2.5) into (2.3), it follows $|\lambda I-S|=$

$$
\begin{aligned}
& =\beta_{k} \prod_{j=1}^{k-1}\left(\beta_{j}-c_{j} \mu_{m_{j}}\left(M_{j}\right)\right)^{n_{j+1}} \prod_{i=1}^{m_{j}-1}\left(\beta_{j}-c_{j} \mu_{i}\left(M_{j}\right)\right)^{n_{j+1}} \\
& =\frac{D_{k}}{D_{k-1}} \prod_{j=1}^{k-1} \frac{D_{j}^{n_{j+1}}}{D_{j-1}^{n_{j+1}}} \prod_{i=1}^{m_{j}-1}\left(\frac{D_{j}}{D_{j-1}}+c_{j} \mu_{m_{j}}\left(M_{j}\right)-c_{j} \mu_{i}\left(M_{j}\right)\right)^{n_{j+1}} \\
& =\frac{D_{k}}{D_{k-1}} \prod_{j=1}^{k-1} \frac{D_{j}^{n_{j+1}}}{D_{j-1}^{n_{j+1}}} \prod_{i=1}^{m_{j}-1}\left(\frac{D_{j}-c_{j} \mu_{i}\left(M_{j}\right) D_{j-1}+c_{j} \mu_{m_{j}}\left(M_{j}\right) D_{j-1}}{D_{j-1}}\right)^{n_{j+1}} \\
& =\frac{D_{k}}{D_{k-1}} \prod_{j=1}^{k-1} \frac{D_{j}^{n_{j+1}}}{D_{j-1}^{n_{j+1}}} \frac{1}{D_{j-1}^{\left(m_{j}-1\right) n_{j+1}}} \prod_{i=1}^{m_{j}-1}\left(D_{j}-c_{j} \mu_{i}\left(M_{j}\right) D_{j-1}+c_{j} \mu_{m_{j}}\left(M_{j}\right) D_{j-1}\right)^{n_{j+1}} \\
& =\frac{D_{k}}{D_{k-1}} \prod_{j=1}^{k-1} \frac{D_{j}^{n_{j+1}}}{D_{j-1}^{n_{j}}} \prod_{i=1}^{m_{j}-1}\left(D_{j}-c_{j} \mu_{i}\left(M_{j}\right) D_{j-1}+c_{j} \mu_{m_{j}}\left(M_{j}\right) D_{j-1}\right)^{n_{j+1}} \\
& =\frac{D_{k}}{D_{k-1}} \prod_{j=1}^{k-1} \frac{D_{j}^{n_{j+1}}}{D_{j-1}^{n_{j}}} \prod_{i=1}^{m_{j}-1}\left(\left(\lambda-\gamma_{j}-c_{j} \mu_{i}\left(M_{j}\right)\right) D_{j-1}-w_{j-1}^{2} m_{j-1} D_{j-2}\right)^{n_{j+1}} \\
& =D_{k} \prod_{j \in \Omega-\Delta}\left(\left(\lambda-\gamma_{j}\right) D_{j-1}-w_{j-1}^{2} m_{j-1} D_{j-2}\right)^{n_{j}-n_{j+1}}
\end{aligned}
$$

$$
\prod_{j \in \Delta} \prod_{i=1}^{m_{j}-1}\left(\left(\lambda-\gamma_{j}-\mu_{i}\left(M_{j}\right)\right) D_{j-1}-w_{j-1}^{2} m_{j-1} D_{j-2}\right)^{n_{j+1}} .
$$


Then, the result is proven for all $\lambda \in \mathbb{R}$ such that $D_{j}(\lambda) \neq 0$, with $j=1, \ldots, k-1$. Now, we consider $\lambda_{0} \in \mathbb{R}$ such that $D_{s}\left(\lambda_{0}\right)=0$ for some $s \in\{1, \ldots, k-1\}$. Since the zeros of any nonzero polynomial are isolated, there exists a neighborhood $N\left(\lambda_{0}\right)$ of $\lambda_{0}$ such that $D_{j}(\lambda) \neq 0$ for all $\lambda \in N\left(\lambda_{0}\right)-\left\{\lambda_{0}\right\}$ and for all $j=1, \ldots, k-1$. Hence, the obtained expression for the characteristic polynomial of $S$ holds for all $\lambda \in N\left(\lambda_{0}\right)-\left\{\lambda_{0}\right\}$. By continuity, taking the limit as $\lambda$ tends to $\lambda_{0}$, we may conclude that this expression is valid for all $\lambda \in \mathbb{R}$.

Definition 2.6. For $j=1, \ldots, k$, let $X_{j}$ be the $j \times j$ leading principal submatrix of the $k \times k$ symmetric tridiagonal matrix $X_{k}=$

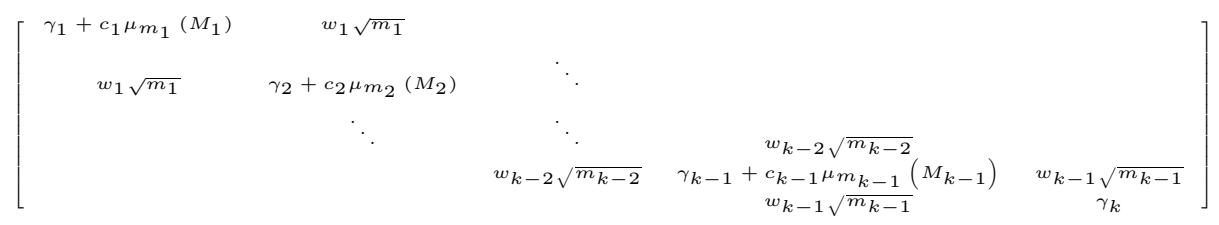

At this point, we recall the well known fact (cf. [12, page 229]) that the characteristic polynomials $T_{j}(\lambda)$ of the $j \times j$ leading principal submatrix of the $k \times k$ symmetric tridiagonal matrix

$$
\left[\begin{array}{ccccc}
a_{1} & b_{1} & & & \\
b_{1} & a_{2} & b_{2} & & \\
& \ddots & \ddots & \ddots & \\
& & \ddots & a_{k-1} & b_{k-1} \\
& & & b_{k-1} & a_{k}
\end{array}\right]
$$

satisfy the three-term recursion formula

$$
T_{j}(\lambda)=\left(\lambda-a_{j}\right) T_{j-1}(\lambda)-b_{j-1}^{2} T_{j-2}(\lambda)
$$

with $T_{0}(\lambda)=1$ and $T_{1}(\lambda)=\lambda-a_{1}$.

The next lemma gives the relationship between the polynomials $D_{j}$ and the matrices $X_{j}$.

Lemma 2.7. For $j=1, \ldots, k$,

$$
\left|\lambda I-X_{j}\right|=D_{j}(\lambda)
$$

where the polynomial $D_{j}(\lambda)$ is as in Definition 2.6.

Proof. Taking into account that

$$
\left|\lambda I-X_{1}\right|=\lambda-\gamma_{1}-c_{1} \mu_{m_{1}}\left(M_{1}\right) \gamma_{1}=D_{1}(\lambda),
$$


let us prove the equality (2.7) by induction, assuming its validity when the index is less than $j$, with $2 \leq j \leq k$. In fact,

$$
\begin{aligned}
& \left|\lambda I-X_{j}\right| \\
& =\left|\begin{array}{cccc}
\lambda-\gamma_{1}-c_{1} \mu_{m_{1}}\left(M_{1}\right) & -w_{1} \sqrt{m_{1}} & & \\
-w_{1} \sqrt{m_{1}} & \lambda-\gamma_{2}-c_{2} \mu_{m_{2}}\left(M_{2}\right) & \ddots & \\
\ddots & \ddots & \ddots & -w_{j-1} \sqrt{m_{j-1}} \\
& & w_{j-1} \sqrt{m_{j-1}} & \lambda-\gamma_{j}-c_{j} \mu_{m_{j}}\left(M_{j}\right)
\end{array}\right| \\
& =\left(\lambda-\gamma_{j}-c_{j} \mu_{m_{j}}\left(M_{j}\right)\right)\left|\lambda I-X_{j-1}\right|-w_{j-1}^{2} m_{j-1}\left|\lambda I-X_{j-2}\right| \text { (according to (2.6)) } \\
& =\left(\lambda-\gamma_{j}-c_{j} \mu_{m_{j}}\left(M_{j}\right)\right) D_{j-1}(\lambda)-w_{j-1}^{2} m_{j-1} D_{j-2}(\lambda) \text { (by induction hypothesis) } \\
& =D_{j}(\lambda) \text {. }
\end{aligned}
$$

From Lemma 2.7, we are able to determine the polynomials $D_{j}$, with $j \in \Omega-\Delta$, which appear in Theorem 2.5. Now, for $j \in \Delta$ and $1 \leq i \leq m_{j}-1$, we consider the polynomials

$$
D_{j, i}(\lambda)=\left(\lambda-\gamma_{j}-\mu_{i}\left(M_{j}\right)\right) D_{j-1}-w_{j-1}^{2} m_{j-1} D_{j-2},
$$

where $m_{0}=0$ and $D_{-1}$ is an arbitrary constant. These polynomials are also factors of the characteristic polynomial of the matrix $S$, as it is stated by Theorem 2.5.

Definition 2.8. For $j \in \Delta$ and $i=1, \ldots, m_{j}-1$, let $X_{j, i}=$

$$
\left[\begin{array}{ccccc}
\gamma_{1}+c_{1} \mu_{m_{1}}\left(M_{1}\right) & w_{1} \sqrt{m_{1}} & & & \\
w_{1} \sqrt{m_{1}} & \ddots & \ddots & & \\
& \ddots & \ddots & w_{j-2} \sqrt{m_{j-2}} & \\
& & w_{j-2} \sqrt{m_{j-2}} & \gamma_{j-1}+c_{j-1} \mu_{m_{j-1}}\left(M_{j-1}\right) & w_{j-1} \sqrt{m_{j-1}} \\
& & & w_{j-1} \sqrt{m_{j-1}} & \gamma_{j}+\mu_{i}\left(M_{j}\right)
\end{array}\right]
$$

The next lemma gives the relationship between the polynomials $D_{j, i}$ and the matrices $X_{j, i}$.

Lemma 2.9. For $j=1, \ldots, k-1$ and $i=1, \ldots, m_{j}-1$

$$
\left|\lambda I-X_{j, i}\right|=D_{j, i}(\lambda),
$$

where the polynomials $D_{j, i}(\lambda)$ are defined in (2.8).

Proof. Taking into account (2.6), the proof is similar to the proof of Lemma 2.7.

From Theorem 2.5 and Lemmas 2.7 and 2.9, we get the main result of this paper.

Theorem 2.10. Consider the $j \times j$ leading principal submatrices $X_{j}$ of the matrix $X_{k}$ in (2.6) and the matrices $X_{j, i}$, according to Definition 2.8, for $j=1, \ldots, k-1$ 
and $i=1, \ldots, m_{j}-1$. Then

$$
\sigma(S)=\sigma\left(X_{k}\right) \cup\left(\cup_{j \in \Omega-\Delta} \sigma\left(X_{j}\right)^{n_{j}-n_{j+1}}\right) \cup\left(\cup_{j \in \Delta} \cup_{i=1}^{m_{j}-1} \sigma\left(X_{j, i}\right)^{n_{j+1}}\right),
$$

where $\sigma\left(X_{j}\right)^{n_{j}-n_{j+1}}$ and $\sigma\left(X_{j, i}\right)^{n_{j+1}}$ mean that each eigenvalue in $\sigma\left(X_{j}\right)$ and in $\sigma\left(X_{j, i}\right)$ must be considered with multiplicity $n_{j}-n_{j+1}$ and $n_{j+1}$, respectively. Furthermore, the multiplicities of equal eigenvalues obtained in different matrices (if any), must be added.

Notice that the symmetric tridiagonal matrices with nonzero co-diagonal entries, which are the case of the matrices $X_{k}, X_{j}$ and $X_{j, i}$, have simple eigenvalues [9].

3. The Laplacian eigenvalues of $\mathcal{B}(\boldsymbol{F})$. Throughout this section, for each $j$, the Laplacian eigenvalues of $\mathcal{G}_{j}$ are denoted by

$$
l_{1}\left(\mathcal{G}_{j}\right), l_{2}\left(\mathcal{G}_{j}\right), \ldots, l_{m_{j}-1}\left(\mathcal{G}_{j}\right), l_{m_{j}}\left(\mathcal{G}_{j}\right)=0 .
$$

Corollary 3.1. If $\mathcal{B}$ is a generalized Bethe tree with $k$ levels and $\mathrm{F}=\left\{\mathcal{G}_{j}: j \in\right.$ $\Delta\}$ is a family of graphs $\mathcal{G}_{j}$ of order $m_{j}$ with indices in a subset of levels $\Delta$, then the spectrum of $L(\mathcal{B}(\mathrm{F}))$ is

$$
\sigma(L(\mathcal{B}(\mathrm{F})))=\sigma\left(U_{k}\right) \cup\left(\cup_{j \in \Omega-\Delta} \sigma\left(U_{j}\right)\right)^{n_{j}-n_{j+1}} \cup\left(\cup_{j \in \Delta} \cup_{i=1}^{m_{j}-1} \sigma\left(U_{j, i}\right)^{n_{j+1}}\right)
$$

where, for $j=1, \ldots, k-1, U_{j}$ is the $j \times j$ leading principal submatrix of the matrix

$$
U_{k}=\left[\begin{array}{ccccc}
\delta_{1} & w_{1} \sqrt{m_{1}} & & & \\
w_{1} \sqrt{m_{1}} & \delta_{2} & \ddots & & \\
& \ddots & \ddots & w_{k-2} \sqrt{m_{k-2}} & \\
& & w_{k-2} \sqrt{m_{k-2}} & \delta_{k-1} & w_{k-1} \sqrt{m_{k-1}} \\
& & & w_{k-1} \sqrt{m_{k-1}} & \delta_{k}
\end{array}\right]
$$

and

$$
U_{j, i}=\left[\begin{array}{ccccc}
\delta_{1} & w_{1} \sqrt{m_{1}} & & & \\
w_{1} \sqrt{m_{1}} & \ddots & \ddots & & \\
& \ddots & \ddots & w_{j-2} \sqrt{m_{j-2}} & \\
& & w_{j-2} \sqrt{m_{j-2}} & \delta_{j-1} & w_{j-1} \sqrt{m_{j-1}} \\
& & & w_{j-1} \sqrt{m_{j-1}} & \delta_{j}+l_{i}\left(\mathcal{G}_{j}\right)
\end{array}\right]
$$

The multiplicities of the eigenvalues of $L(\mathcal{B}(\mathrm{F}))$ must be considered as in Theorem 2.10.

Proof. The Laplacian matrix of $\mathcal{B}(F)$ is the matrix $S$, with $\gamma_{j}=\delta_{j}(1 \leq j \leq k)$,

$$
S_{j}=\left\{\begin{array}{ll}
\delta_{j} I_{m_{j}}+L\left(\mathcal{G}_{j}\right) & \text { if } j \in \Delta \\
\delta_{j} I_{m_{j}} & \text { otherwise }
\end{array},\right.
$$


and $L\left(\mathcal{G}_{j}\right) \mathbf{e}_{m_{j}}=\mathbf{0}=0 \mathbf{e}_{m_{j}}$. Then, the spectrum of $L(\mathcal{B}(F))$ is given by Theorem 2.10, replacing the matrices $X_{k}, X_{j}$, and $X_{i, j}$ by the matrices $U_{k}, U_{j}$, and $U_{j, i}$, respectively.

We recall that $\mathcal{P}_{m}(u), \mathcal{S}_{m}(u), \mathcal{C}_{m}(u)$, and $\mathcal{K}_{m}(u)$ are the path, star, cycle, and complete graph on $m$ vertices, respectively, where the edges have weight equal to $u$. The Laplacian eigenvalues of these graphs are:

Laplacian eigenvalues

$$
\begin{array}{cc}
\mathcal{P}_{m}(u): & u\left(2+2 \cos \frac{\pi i}{m}\right), 1 \leq i \leq m \\
\mathcal{S}_{m}(u): & u m, u, \ldots, u, 0 \\
\mathcal{C}_{m}(u): & u\left(2-2 \cos \frac{2 \pi i}{m}\right), 1 \leq i \leq m \\
\mathcal{K}_{m}(u): & u m, \ldots, u m, 0
\end{array}
$$

EXAMPLE 3.2. For the graph $\mathcal{B}(F)$ depicted in Figure 1.1, we have $k=4$, $d_{1}=1, d_{2}=5, d_{3}=3, d_{4}=2, n_{1}=16, n_{2}=4, n_{3}=2, n_{4}=1$, and $\Omega=\{1,2,3\}$. Moreover, $\Delta=\{1,3\}, m_{1}=4, m_{2}=2, m_{3}=2, \mathcal{G}_{1}=\mathcal{S}_{4}\left(u_{1}\right)$, and $\mathcal{G}_{3}=\mathcal{P}_{2}\left(u_{3}\right)$. We have $l_{1}\left(\mathcal{G}_{1}\right)=4 u_{1}, l_{2}\left(\mathcal{G}_{1}\right)=l_{3}\left(\mathcal{G}_{1}\right)=u_{1}$, and $l_{1}\left(\mathcal{G}_{3}\right)=2 u_{3}$. From Corollary 3.1,

$$
\sigma(L(\mathcal{B}(F)))=\sigma\left(U_{4}\right) \cup \sigma\left(U_{2}\right)^{4-2} \cup \sigma\left(U_{1,1}\right)^{4} \cup \sigma\left(U_{1,2}\right)^{4} \cup \sigma\left(U_{1,3}\right)^{4} \cup \sigma\left(U_{3,1}\right)^{1},
$$

where

$$
\begin{aligned}
& U_{4}=\left[\begin{array}{cccc}
w_{1} & 2 w_{1} & & \\
2 w_{1} & 4 w_{1}+w_{2} & \sqrt{2} w_{2} & \\
& \sqrt{2} w_{2} & 2 w_{2}+w_{3} & \sqrt{2} w_{3} \\
& & \sqrt{2} w_{3} & 2 w_{3}
\end{array}\right] \\
& U_{2}=\left[\begin{array}{cc}
w_{1} & 2 w_{1} \\
2 w_{1} & 4 w_{1}+w_{2}
\end{array}\right], \\
& U_{1,1}=\left[w_{1}+l_{1}\left(\mathcal{G}_{1}\right)\right]=\left[w_{1}+4 u_{1}\right], \\
& U_{1,2}=\left[w_{1}+l_{2}\left(\mathcal{G}_{1}\right)\right]=\left[w_{1}+u_{1}\right]=U_{1,3}=\left[1+l_{3}\left(\mathcal{G}_{1}\right)\right],
\end{aligned}
$$

and

$$
\begin{aligned}
& U_{3,1}=\left[\begin{array}{ccc}
w_{1} & 2 w_{1} & \\
2 w_{1} & 4 w_{1}+w_{2} & \sqrt{2} w_{2} \\
& \sqrt{2} w_{2} & 2 w_{2}+w_{3}+l_{1}\left(\mathcal{G}_{3}\right)
\end{array}\right] \\
& =\left[\begin{array}{ccc}
w_{1} & 2 w_{1} & \\
2 w_{1} & 4 w_{1}+w_{2} & \sqrt{2} w_{2} \\
& \sqrt{2} w_{2} & 2 w_{2}+w_{3}+2 u_{3}
\end{array}\right] \text {. }
\end{aligned}
$$


If $w_{1}=2, w_{2}=2.5, w_{3}=3, u_{1}=1.5$, and $u_{3}=2$, then the eigenvalues of $L(\mathcal{B}(F))$, with four decimal places, are:

$\begin{array}{cccccc} & & & & & \text { multiplicity } \\ U_{2}: & 0.4137 & 12.0863 & & & n_{2}-n_{3}=2 \\ U_{4}: & 0 & 2.5150 & 9.4153 & 14.5697 & 1 \\ U_{1,1}: & 8 & & & & n_{2}=4 \\ U_{1,2}: & 3.5 & & & & n_{2}=4 \\ U_{1,3}: & 3.5 & & & & n_{2}=4 \\ U_{3,1}: & 0.2568 & 8.8567 & 15.3864 & n_{4}=1\end{array}$

4. The eigenvalues of the signless Laplacian and adjacency matrices of $\mathcal{B}(\boldsymbol{F})$. If every vertex of $\mathcal{G}$ has degree $r$, then $\mathcal{G}$ is called a regular graph of degree $r$. Throughout this section, we consider a generalized Bethe tree $\mathcal{B}$ with $k$ levels and a family of graphs $F=\left\{\mathcal{G}_{j}: j \in \Delta\right\}$ and then we apply Theorem 2.10 to find the eigenvalues of $Q(\mathcal{B}(F))$ and $A\left(\mathcal{B}(F)\right.$ ), whenever $\mathcal{G}_{j}$ is a regular graph of order $m_{j}$, with $j \in \Delta$.

For $j \in \Delta$, we assume that $\mathcal{G}_{j}$ is a regular graph of degree $r_{j}$ and order $m_{j}$.

EXAMPLE 4.1. The graph depicted in Figure 4.1 is the graph $\mathcal{B}(F)$, with $F=$ $\left\{\mathcal{G}_{1}=2 \mathcal{P}_{2}\left(u_{1}\right), \mathcal{G}_{4}=\mathcal{C}_{3}\left(u_{3}\right)\right\}$.

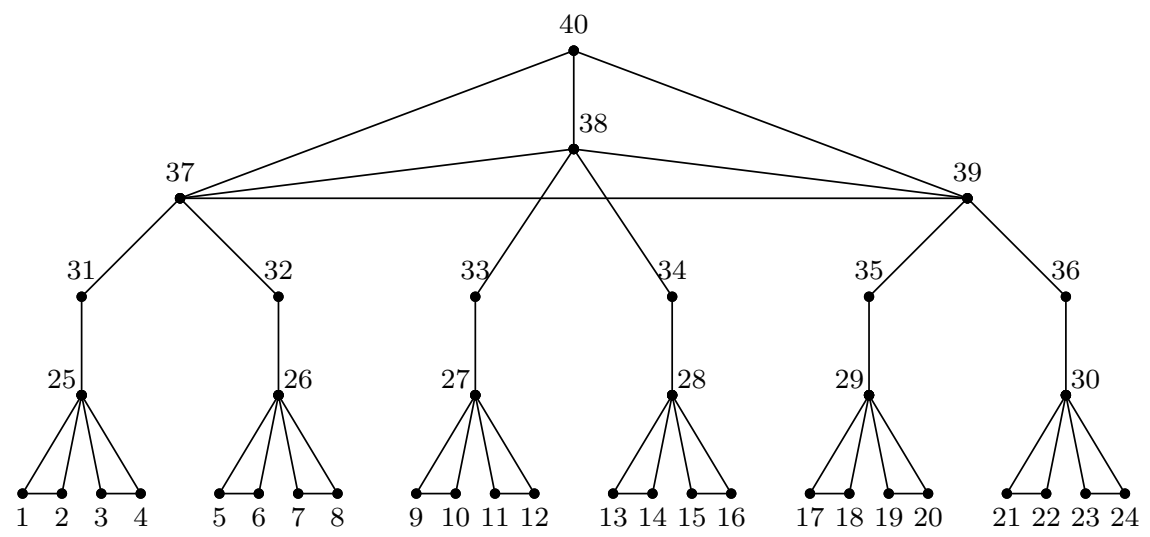

FIG. 4.1. The graph $\mathcal{B}(\mathrm{F})$, with $\mathrm{F}=\left\{\mathcal{G}_{1}=2 \mathcal{P}_{2}\left(u_{1}\right), \mathcal{G}_{4}=\mathcal{C}_{3}\left(u_{3}\right)\right\}$.

Using the labelling for the vertices of $\mathcal{B}(F)$ as in the graph of Figure 1.1, that is, from the pendant vertices to the root and, in each level, from the left to the right, 
the signless Laplacian and adjacency matrices of $\mathcal{B}(F)$ are

$$
Q(\mathcal{B}(F))=\left[\begin{array}{ccccc}
I_{n_{2}} \otimes Q_{1} & I_{n_{2}} \otimes \mathbf{e}_{m_{1}} & & & \\
I_{n_{2}} \otimes \mathbf{e}_{m_{1}}^{T} & \ddots & \ddots & & \\
& \ddots & I_{n_{k-1}} \otimes Q_{k-2} & I_{n_{k-1}} \otimes \mathbf{e}_{m_{k-2}} & \\
& & I_{n_{k-1}} \otimes \mathbf{e}_{m_{k-2}}^{T} & Q_{k-1} & \mathbf{e}_{m_{k-1}} \\
& & & \mathbf{e}_{m_{k-1}}^{T} & \delta_{k}
\end{array}\right]
$$

where $Q_{j}=\left\{\begin{array}{ll}\delta_{j} I_{m_{j}}+Q\left(\mathcal{G}_{j}\right) & \text { if } j \in \Delta \\ \delta_{j} I_{m_{j}} & \text { otherwise }\end{array}\right.$, and

$$
A(\mathcal{B}(F))=\left[\begin{array}{ccccc}
I_{n_{2}} \otimes A_{1} & I_{n_{2}} \otimes \mathbf{e}_{m_{1}} & & & \\
I_{n_{2}} \otimes \mathbf{e}_{m_{1}}^{T} & \ddots & \ddots & & \\
& \ddots & I_{n_{k-1}} \otimes A_{k-2} & I_{n_{k-1}} \otimes \mathbf{e}_{m_{k-2}} & \\
& & I_{n_{k-1}} \otimes \mathbf{e}_{m_{k-2}}^{T} & A_{k-1} & \mathbf{e}_{m_{k-1}} \\
& & & \mathbf{e}_{m_{k-1}}^{T} & 0
\end{array}\right]
$$

where $A_{j}=\left\{\begin{array}{ll}A\left(\mathcal{G}_{j}\right) & \text { if } j \in \Delta \\ 0 & \text { otherwise }\end{array}\right.$.

Let us consider a regular graph $\mathcal{G}$ of degree $r$ and order $m$, and let us denote its signless Laplacian eigenvalues by

$$
q_{1}(\mathcal{G}), q_{2}(\mathcal{G}), \ldots, q_{m-1}(\mathcal{G}), q_{m}(\mathcal{G})
$$

and its adjacency eigenvalues by

$$
\lambda_{1}(\mathcal{G}), \lambda_{2}(\mathcal{G}), \ldots, \lambda_{m-1}(\mathcal{G}), \lambda_{m}(\mathcal{G}) .
$$

Assuming that the edges of $\mathcal{G}$ have a weight equal to $u$, then

$$
Q(\mathcal{G}) \mathbf{e}_{m}=2 r u \mathbf{e}_{m} \text { and } A(\mathcal{G}) \mathbf{e}_{m}=r u \mathbf{e}_{m},
$$

and we may write $\lambda_{m}(\mathcal{G})=r u$ and $q_{m}(\mathcal{G})=2 r u$.

Corollary 4.2. If, for each $j \in \Delta$, the graph $\mathcal{G}_{j}$ is a regular graph of degree $r_{j}$, then the spectrum of $Q(\mathcal{B}(\mathrm{F}))$ is

$$
\sigma(Q(\mathcal{B}(\mathrm{F})))=\sigma\left(V_{k}\right) \cup\left(\cup_{j \in \Omega-\Delta} \sigma\left(V_{j}\right)^{n_{j}-n_{j+1}}\right) \cup\left(\cup_{j \in \Delta} \cup_{i=1}^{m_{j}-1} \sigma\left(V_{j, i}\right)^{n_{j+1}}\right)
$$

where, for $j=1, \ldots, k-1, V_{j}$ is the $j \times j$ leading principal submatrix of

$$
V_{k}=\left[\begin{array}{ccccc}
\delta_{1}+2 c_{1} u_{1} r_{1} & w_{1} \sqrt{m_{1}} & & & \\
w_{1} \sqrt{m_{1}} & \ddots & \ddots & & \\
& \ddots & \ddots & w_{k-2} \sqrt{m_{k-2}} & \\
& & w_{k-2} \sqrt{m_{k-2}} & \delta_{k-1}+2 c_{k-1} u_{k-1} r_{k-1} & w_{k-1} \sqrt{m_{k-1}} \\
& & & w_{k-1} \sqrt{m_{k-1}} & \delta_{k}
\end{array}\right],
$$




$$
V_{j, i}=\left[\begin{array}{ccccc}
\delta_{1}+2 c_{1} u_{1} r_{1} & w_{1} \sqrt{m_{1}} & & & \\
w_{1} \sqrt{m_{1}} & \ddots & \ddots & & \\
& \ddots & \ddots & w_{j-2} \sqrt{m_{j-2}} & \\
& & w_{j-2} \sqrt{m_{j-2}} & \delta_{j-1}+2 c_{j-1} u_{j-1} r_{j-1} & w_{j-1} \sqrt{m_{j-1}} \\
& & & w_{j-1} \sqrt{m_{j-1}} & \delta_{j}+q_{i}\left(\mathcal{G}_{j}\right)
\end{array}\right]
$$

and the multiplicities of the eigenvalues of $Q(\mathcal{B}(\mathrm{F}))$ are as in Theorem 2.10.

Proof. The signless Laplacian matrix of $\mathcal{B}(F)$ is the matrix $S$, with $\gamma_{i}=\delta_{i}$, for $i=1, \ldots, k$, and $M_{j}=Q\left(\mathcal{G}_{j}\right)$ if $j \in \Delta$ and it is the zero matrix otherwise. Moreover, $Q\left(\mathcal{G}_{j}\right) \mathbf{e}_{m_{j}}=2 u_{j} r_{j} \mathbf{e}_{m_{j}}$. Then, the result follows directly from Theorem 2.10.

EXAmPLE 4.3. For the graph $\mathcal{B}(F)$ depicted in Figure $4.1, \mathcal{B}=\mathcal{B}_{5}(1,5,2,3,3)$ and we have $k=5, \delta_{1}=w_{1}, \delta_{2}=4 w_{1}+w_{2}, \delta_{3}=w_{2}+w_{3}, \delta_{4}=2 w_{3}+w_{4}, \delta_{5}=3 w_{4}$, $n_{1}=24, n_{2}=n_{3}=6, n_{4}=3$, and $n_{5}=1$. Moreover, $\Omega=\{1,3,4\}, \Delta=\{1,4\}$, $m_{1}=4, m_{2}=1, m_{3}=2, m_{4}=3, \mathcal{G}_{1}=2 \mathcal{P}_{2}\left(u_{1}\right), r_{1}=1, \mathcal{G}_{4}=\mathcal{C}_{3}\left(u_{4}\right)$ and $r_{4}=2$. Since $q_{1}\left(\mathcal{G}_{1}\right)=2 u_{1}, q_{2}\left(\mathcal{G}_{1}\right)=q_{3}\left(\mathcal{G}_{1}\right)=0, q_{1}\left(\mathcal{G}_{4}\right)=q_{2}\left(\mathcal{G}_{4}\right)=u_{4}$, from Corollary 4.2 , it follows

$\sigma(Q(\mathcal{B}(F)))=\sigma\left(V_{5}\right) \cup \sigma\left(V_{3}\right)^{6-3} \cup \sigma\left(V_{1,1}\right)^{6} \cup \sigma\left(V_{1,2}\right)^{6} \cup \sigma\left(V_{1,3}\right)^{6} \cup \sigma\left(V_{4,1}\right)^{1} \cup \sigma\left(V_{4,2}\right)^{1}$,

where

$$
\begin{aligned}
& V_{5}=\left[\begin{array}{ccccc}
\delta_{1}+2 u_{1} & 2 w_{1} & & & \\
2 w_{1} & \delta_{2} & w_{2} & & \\
& w_{2} & \delta_{3} & w_{3} \sqrt{2} & \\
& & w_{3} \sqrt{2} & \delta_{4}+4 u_{4} & w_{4} \sqrt{3} \\
& & & w_{4} \sqrt{3} & \delta_{5}
\end{array}\right] \\
& V_{3}=\left[\begin{array}{ccc}
\delta_{1}+2 u_{1} & 2 w_{1} & \\
2 w_{1} & \delta_{2} & w_{2} \\
& w_{2} & \delta_{3}
\end{array}\right] \\
& V_{1,1}=\left[\delta_{1}+2 u_{1}\right], V_{1,2}=V_{1,3}=\left[\delta_{1}\right],
\end{aligned}
$$

and

$$
V_{4,1}=V_{4,2}=\left[\begin{array}{cccc}
\delta_{1}+2 u_{1} & 2 w_{1} & & \\
2 w_{1} & \delta_{2} & w_{2} & \\
& w_{2} & \delta_{3} & w_{3} \sqrt{2} \\
& & w_{3} \sqrt{2} & \delta_{4}+u_{4}
\end{array}\right]
$$


Setting $w_{1}=2, w_{2}=1.5, w_{3}=2.5, w_{4}=3, u_{1}=1.8$, and $u_{4}=2.2$, the eigenvalues of $Q(\mathcal{B}(F)$ ), with four decimal places, are:

\begin{tabular}{|c|c|c|c|c|c|c|}
\hline$V_{3}:$ & 2.5897 & 4.3113 & 12.1990 & & & $\begin{array}{l}\text { multiplicity } \\
n_{3}-n_{4}=3\end{array}$ \\
\hline$V_{5}:$ & 2.0698 & 3.5867 & 7.0275 & 12.1785 & 20.0374 & 1 \\
\hline$V_{1,1}:$ & 5.6 & & & & & $n_{2}=6$ \\
\hline$V_{1,2}:$ & 2 & & & & & $n_{2}=6$ \\
\hline$V_{1,3}:$ & 2 & & & & & $n_{2}=6$ \\
\hline$V_{4,1}:$ & 1.8425 & 3.5046 & 11.4279 & 12.5250 & & $n_{5}=1$ \\
\hline$V_{4,2}:$ & 1.8425 & 3.5046 & 11.4279 & 12.5250 & & $n_{5}=1$ \\
\hline
\end{tabular}

COROLlary 4.4. If, for each $j \in \Delta$, the graph $\mathcal{G}_{j}$ is regular of degree $r_{j}$, then the spectrum of $A(\mathcal{B}(\mathrm{F}))$ is

$$
\sigma(A(\mathcal{B}(\mathrm{F})))=\sigma\left(W_{k}\right) \cup\left(\cup_{j \in \Omega-\Delta} \sigma\left(W_{j}\right)^{n_{j}-n_{j+1}}\right) \cup\left(\cup_{j \in \Delta} \cup_{i=1}^{m_{j}-1} \sigma\left(W_{j, i}\right)^{n_{j+1}}\right),
$$

where, for $j=1, \ldots, k-1, X_{j}$ is the $j \times j$ leading principal submatrix of the $k \times k$ symmetric tridiagonal matrix

$$
W_{k}=\left[\begin{array}{ccccc}
c_{1} u_{1} r_{1} & w_{1} \sqrt{m_{1}} & & & \\
w_{1} \sqrt{m_{1}} & c_{2} u_{2} r_{2} & \ddots & & \\
& \ddots & \ddots & w_{k-2} \sqrt{m_{k-2}} & \\
& & w_{k-2} \sqrt{m_{k-2}} & c_{k-1} u_{k-1} r_{k-1} & w_{k-1} \sqrt{m_{k-1}} \\
& & & w_{k-1} \sqrt{m_{k-1}} & 0
\end{array}\right]
$$

and

$$
W_{j, i}=\left[\begin{array}{ccccc}
c_{1} u_{1} r_{1} & w_{1} \sqrt{m_{1}} & & & \\
w_{1} \sqrt{m_{1}} & \ddots & \ddots & & \\
& \ddots & \ddots & w_{j-2} \sqrt{m_{j-2}} & \\
& & w_{j-2} \sqrt{m_{j-2}} & c_{j-1} u_{j-1} r_{j-1} & w_{j-1} \sqrt{m_{j-1}} \\
& & & w_{j-1} \sqrt{m_{j-1}} & \lambda_{i}\left(\mathcal{G}_{j}\right)
\end{array}\right],
$$

and the multiplicities of the eigenvalues of $A(\mathcal{B}(\mathrm{F}))$ are as in Theorem 2.10.

Proof. The adjacency matrix of $\mathcal{B}(F)$ is the matrix $S$, with $\gamma_{i}=0(1 \leq i \leq k)$ and $M_{j}=A\left(\mathcal{G}_{j}\right)$ if $j \in \Delta$ and it is the zero matrix otherwise. Moreover, $A\left(\mathcal{G}_{j}\right) \mathbf{e}_{m_{j}}=$ $u_{j} r_{j} \mathbf{e}_{m_{j}}$. Then the result follows directly from Theorem 2.10.

In the next example, we look for the eigenvalues of the adjacency matrix $A(\mathcal{B}(F))$ of the graph depicted in Figure 4.1. 
EXAMPLE 4.5. Consider the graph $\mathcal{B}(F)$ depicted in Figure 4.1. Then we have $\lambda_{1}\left(\mathcal{G}_{1}\right)=u_{1}, \lambda_{2}\left(\mathcal{G}_{1}\right)=\lambda_{3}\left(\mathcal{G}_{1}\right)=-u_{1}, \lambda_{1}\left(\mathcal{G}_{4}\right)=\lambda_{2}\left(\mathcal{G}_{4}\right)=-u_{4}$ and, applying Corollary 4.4

$\sigma(A(\mathcal{B}(F)))=\sigma\left(W_{5}\right) \cup \sigma\left(W_{3}\right)^{6-3} \cup \sigma\left(W_{1,1}\right)^{6} \cup \sigma\left(W_{1,2}\right)^{6} \cup \sigma\left(W_{1,3}\right)^{6} \cup \sigma\left(W_{4,1}\right)^{1} \cup \sigma\left(W_{4,2}\right)^{1}$,

where

$$
\begin{aligned}
& W_{5}=\left[\begin{array}{ccccc}
u_{1} & 2 w_{1} & & & \\
2 w_{1} & 0 & w_{2} & & \\
& w_{2} & 0 & w_{3} \sqrt{2} & \\
& & w_{3} \sqrt{2} & 2 u_{4} & w_{4} \sqrt{3} \\
& & & w_{4} \sqrt{3} & 0
\end{array}\right], \\
& W_{3}=\left[\begin{array}{ccc}
u_{1} & 2 w_{1} & \\
2 w_{1} & 0 & w_{2} \\
& w_{2} & 0
\end{array}\right] \\
& W_{1,1}=\left[u_{1}\right], W_{1,2}=W_{1,3}=\left[-u_{1}\right],
\end{aligned}
$$

and

$$
W_{4,1}=W_{4,2}=\left[\begin{array}{cccc}
u_{1} & 2 w_{1} & & \\
2 w_{1} & 0 & w_{2} & \\
& w_{2} & 0 & w_{3} \sqrt{2} \\
& & w_{3} \sqrt{2} & -u_{4}
\end{array}\right]
$$

Setting $w_{1}=2, w_{2}=1.5, w_{3}=2.5, w_{4}=3, u_{1}=1.8$ and $u_{4}=2.2$, the eigenvalues of $A(\mathcal{B}(F))$ with four decimal places, are:

$\begin{array}{ccccccc}W_{3}: & -3.5930 & 0.2178 & 5.1752 & & & n_{3}-n_{4}=3 \\ W_{5}: & -4.7075 & -3.2592 & 0.1567 & 5.1142 & 8.8959 & 1 \\ W_{1,1}: & 1.8 & & & & & n_{2}=6 \\ W_{1,2}: & -1.8 & & & & n_{2}=6 \\ W_{1,3}: & -1.8 & & & & n_{2}=6 \\ W_{4,1}: & -5.1067 & -3.0782 & 2.5281 & 5.2569 & & n_{5}=1 \\ W_{4,2}: & -5.1067 & -3.0782 & 2.5281 & 5.2569 & & n_{5}=1\end{array}$

Acknowledgment. O. Rojo thanks the hospitality of the Departamento de Matemtica, Universidade de Aveiro, Aveiro, Portugal, and its Center for Research and Development in Mathematics and Applications, where this research was conducted. 


\section{REFERENCES}

[1] D.M. Cardoso, D. Cvetković, P. Rowlinson, and S.K. Simić. A sharp lower bound for the least eigenvalue of the signless Laplacian of a non-bipartite graph. Linear Algebra Appl., 429:2770-2780, 2008.

[2] D. Cardoso, E.A. Martins, M. Robbiano, and V. Trevisan. Computing the Laplacian spectra of some graphs. Discrete Appl. Math., to appear, DOI: 10.1016/j.dam.2011.04.002, 2011.

[3] D. Cvetković, P. Rowlinson, and S.K. Simić. Signless Laplacian of finite graphs. Linear Algebra Appl., 423:155-171, 2007.

[4] D. Cvetković and S.K. Simić. Towards a spectral theory of graphs based on the signless Laplacian I. Publ. Inst. Math. (Beograd) (N.S.), 85(99):19-33, 2009.

[5] D. Cvetković and S.K. Simić. Towards a spectral theory of graphs based on the signless Laplacian II. Linear Algebra Appl., 432:2257-2272, 2010.

[6] D. Cvetković and S.K. Simić. Towards a spectral theory of graphs based on the signless Laplacian III. Appl. Anal. Discrete Math., 4:156-166, 2010.

[7] R. Fernandes, H. Gomes, and E.A. Martins. On the spectra of some graphs like weighted rooted trees. Linear Algebra Appl., 428:2654-2674, 2008.

[8] M. Fiedler. Algebraic connectivity of graphs. Czechoslovak Math. J., 23:298-305, 1973.

[9] G.H. Golub and C.F. Van Loan. Matrix Computations, 2nd edition. John Hopkins University Press, Baltimore, 1989.

[10] R. Merris. Laplacian matrices of graphs: A survey. Linear Algebra Appl., 197/198:143-176, 1994.

[11] O. Rojo and L. Medina. Spectral characterization of some weighted rooted graphs with cliques. Linear Algebra Appl., 433:1388-1409, 2010.

[12] L.N. Trefethen and D. Bau, III. Numerical Linear Algebra. Society for Industrial and Applied Mathematics (SIAM), Philadelphia, 1997.

[13] F. Zhang. Matrix Theory. Springer-Verlag, New York, 1999. 